\title{
Research progress of hyperuricemia on kidney damage
}

\author{
Chonin, Cheang ${ }^{1 \mathrm{st}, *}$, Wengtong, Chan ${ }^{1 \mathrm{st}}$ \\ ${ }^{1}$ Jinan University, College of basic medicine and public health, 510665 Guangzhou Guangdong, China \\ Both of them are co first authors
}

\begin{abstract}
Urea is the main product secreted in normal human urine, which will contain a small amount of uric acid.Uric acid is the end product of purines known as trioxypurines, which have a weakly acidic alcoholic form, and if purines are metabolized, the risk of hyperuricemia will be increased. It has been shown that the risk of developing CKD in patients with increased serum uric acid levels is 2.21 times that of normal patients. There are experimental data to divide patients into two groups, one is patients with hyperuricemia and the other is patients with normal serum uric acid, the results of the study show that patients with hyperuricemia have a significantly greater probability of developing acute kidney injury than patients with normal serum uric acid, and most of them need kidney replacement therapy. This review focuses on the development of kidney damage in hyperuricemia.
\end{abstract}

\section{INTRODUCTION}

High uric acid can deposit in joints, kidneys, stimulate the blood vessel wall, leading to low glucose utilization, and then promote arthritis, uremia, and many other diseases. It has been shown that people with high uric acid have a significantly higher probability of developing hypertension, type 2 diabetes mellitus, stroke, and kidney failure than the general population [1-2]. In recent years, many people have the habit of regular physical examination, and most of the values of uric acid will have an abnormality, which indicates that uric acid is high when the value exceeds four hundred in men and two in women [3]. And in today's population, one in ten people will have high uric acid, so high uric acid is also known as the new fourth high. Hyperuricemic nephropathy is clinically divided into acute and chronic hyperuricemic nephropathy, and the two differ in pathogenesis. Myeloproliferative disorders such as leukemia, lymphoma, and high rates of malignancy, particularly when undergoing radiotherapy and chemotherapy, have large amounts of uric acid that undergo excretion by the kidneys, and uric acid crystals are found in the tubules, causing increased intraluminal pressure in the tubules and increased intracystic pressure in the glomeruli, resulting in a dramatic decrease in the glomerular filtration rate. This study is aimed at the occurrence mechanism of hyperuricemia damaging kidney, and the above emerging prevalent diseases are just on the way to illustrate that hyperuricemia is an important factor affecting kidney health [4-5].

With the improvement of the quality of life of our people, as well as the change of dietary structure, especially the high intake of protein - and purine containing foods, the incidence of hyperuricemia is also gradually increasing. There are approximately $1300 \mathrm{mg}$ of uric acid in a normal person that is newly generated about $650 \mathrm{mg}$ per day while excreting $650 \mathrm{mg}$ at the same time, in a state of balance. If too much uric acid is produced in the body for excretion, or the uric acid excretion function is degraded, it leads to too much retention of uric acid in the body, resulting in the body fluids becoming acidic, affecting the normal function of human cells. If left untreated for a prolonged period of time, gout can be triggered. Second, too fatigue or not enough rest can also lead to a relatively slow metabolism, contributing to the pathogenesis of gout. The amount of newly formed and excreted uric acid is approximately equal in the normal human body on a daily basis, with respect to the amount produced, one-third through food intake, two-thirds through self synthesis in the body, one-third through the excretion pathway, one-third through the intestine, and two-thirds through the kidneys.

\section{Causes of hyperuricemia}

Hyperuricemia can be divided into primary hyperuricemia and secondary hyperuricemia, and when uric acid is too high in humans, the flow rate of blood in the body slows, even the inability to flow, and the metabolism in the body decreases, leading to hoarding of both energy and fat ingested in the body [6] and, in turn, to obesity.

\subsection{Reduced kidney function}

Too high uric acid can lead to the occurrence of kidney disease, and vice versa when the kidney problems are high, uric acid between the kidneys is reciprocal. This is

\footnotetext{
* Corresponding author: zhengjunxianalex@gmail.com
} 
because uric acid is filtered through the blood through the kidneys and then excreted in the urine, so if kidney function declines at a time when the filtered uric acid in the blood is not better excreted, it will lead to an increase in the. The incidence groups of uric acid are all in the middle-aged elderly because of increasing age, slowing down of the function of body mechanisms, progressive decline of kidney function, and higher uric acid [7].

\subsection{Alcohol abuse}

Excessive alcohol consumption not only causes paralyzed nerves and is prone to traffic accidents, but is also one of the factors responsible for high uric acid. Because after alcohol enters the body, the liver is needed to aid metabolism, but when the liver metabolizes alcohol to acetic acid, there is a conflicting relationship with the metabolism of uric acid in the blood, then there is an effect on the excretion of uric acid, so there is a concomitant increase in blood uric acid.

\subsection{Dietary issues}

Uric acid is a weak acid produced by the body when it comes to foods containing purine nucleotides during metabolism and then enters the bloodstream, so if you consume some foods high in purines, such as animal innards, anchovy fish, beans, etc., the human body will metabolize a large amount of uric acid and the concentration of uric acid will become higher.

\subsection{Increased uric acid production}

Patients were defined as having overproduction of uric acid in their urine greater than $500 \mathrm{mg}$ when prescribed more than a low purine diet. The mechanism of the effect may be related to mutations in the enzyme genes, in the process of uric acid production, the number and activity of some enzymes that play a promoting role are increased compared with the activity and the number and activity of related enzymes that play an inhibiting role. During the course of treatment of patients with leukemia, multiple myeloma, and lymphoma with chemotherapy, it results in accelerated metabolism of nucleic acids by leukocytes, triggering secondary hyperuricemia [8-9]. For most patients with primary hyperuricemia, hyperuricemia is caused more by decreased uric acid secretion, and it is mainly caused by decreased tubular secretion, or decreased glomerular filtration.But the specific mechanism is not very clear at present. There is also a condition in which hyperuricemia is secondary to other conditions, such as kidney lesions or myelodysplastic syndrome, or in which elevated uric acid occurs as a result of certain drugs that cause abnormal uric acid metabolism.

\section{Pathogenesis of kidney damage by hyperuricemia}

The onset of uric acid nephropathy is more insidious, patients are asymptomatic when physical mechanisms have changed, and the effects on kidney function become more significant over time [10-12]. The initial clinical features are an increase in uric acid, polymorphic crystals in the urine, leading to the appearance of hematuria and a small amount of proteinuria, accompanied by uremic symptoms such as flank pain, nausea, vomiting, etc. long-term increased urinary acidity produces uric acid crystals and initiates a chronic inflammatory response will reverse the cells of uric acid crystals, activate the complement system and inflammatory cells, and at the same time lead to the release of cytokines and other mediators, which in turn leads to chronic inflammatory damage to the tubulointerstitium. When the concentration of uric acid in the blood reaches a certain saturation state, it will deposit in the kidney because of the crystallization of uric acid formation, which can cause problems in the kidney, such as kidney stones and so on. And hyperuricemia has several kidney factors, such as: urate deposition in kidney tubules, increased fibrosis, and also urate can trigger obstructive nephropathy through stone formation. A concentration of uric acid in the blood that exceeds the normal range is hyperuricemia, usually due to various causes associated with increased production or impaired excretion of uric acid. For example, impaired kidney function causes decreased excretion of uric acid, which leads to failure to excrete uric acid out of the body, high levels of uric acid, and hyperuricemia.

\section{Pathology}

The pathological characteristics of chronic hyperuricemic nephropathy are mainly that uric acid crystals will appear precipitated in the kidney interstitium, especially in the medullary and papillary regions of the kidney, and the state of urate deposition within the kidney parenchyma is evident by microscopy. Chronic hyperuricemic nephropathy is also called gouty nephropathy, which is a deep deposition in the corticomedullary junction, the medulla;Patients with a long history of gout disease whose kidneys show not only tophus formation but also fibrogenesis, glomerulosclerosis, arteriosclerosis, and arterial wall thickening develop gout mainly because of consumption of foods with high purine content. At the time of the gout attack, the patient's condition worsened and his kidney function became lesional because of the absence of timely and effective control of uric acid values in the body [13]. kidney pathology in hyperuricemic nephropathy is characterised by low back acidity, physical weakness and mild proteinuria. Hyperuricemic nephropathy may present with uric acid crystals in the site of ureter and kidney pelvis within a short period of time, slight proteinuria may occur in urine, and when acute, oliguria and anuria may occur, which, if untreated, 
may even lead to diseases such as fibrosis and arteriosclerosis in the kidney tubules.

\section{Triggered elucidation of associated diseases}

Hyperuricemia polydactyly refers to the high level of uric acid in the human body, which can be affected by many factors, such as genetic factors, gender situation and dietary habits, etc. [14]. Once the attack, the disease will cause a huge psychological burden to the patient, when the serum uric acid value of the patient is high to a certain extent, some patients will develop gouty arthritis or gouty kidney damage, arthritis is mostly manifested as erythematous and hot pain in the large joints of the lower limbs, can recur without intervention, especially the hyperuricemia triggered arthritis, gout and so on would be very uncomfortable. Hyperuricemia is fundamental to, but not sufficient to cause, gout, which appears only if urate is deposited in the body's tissues causing damage. The following are relevant conditions that may be triggered after the appearance of hyperuricemia:

\subsection{Gout}

The pathogenetic basis of gout is hyperuricemia, in which more urate is deposited in the body and causes more damage to the body, symptoms of gout may appear. In general, the higher the value of serum uric acid level, the greater the likelihood of developing gout.

\subsection{Arthritis}

Hyperuricemia will show different degrees of joint inflammation, joint pain, swelling, fever and other symptoms are more obvious, the higher uric acid, the more bad joint inflammation.

\subsection{Hypertension}

Multiple epidemiological studies have shown that the level of uric acid has a relationship with hypertension, and that serum uric acid is one of the risk factors for the development of hypertension, and a higher serum uric acid level is associated with a higher risk of developing hypertension. Clinical studies show that $90 \%$ of essential hypertension has high uric acid.

\subsection{Diabetes}

Long term high uric acid triggers the destruction of pancreatic function, which induces the generation of diabetes, and long-term high uric acid is causally related to the onset of diabetes.

\subsection{Metabolic syndrome}

Hyperuricemia can be combined with the generation of metabolic syndrome, which is a further increase in uric acid production resulting from insulin resistance in the body, thus aggravating the problem of hyperuricemia.

\section{6 kidney impairment}

Further deposition of uric acid crystals in the body can lead to kidney artery, chronic inflammation and so on triggering kidney damage, which can lead to chronic kidney disease. Therefore, patients with high uric acid all have relatively weak kidney function.

\section{Interventions for hyperuricemia}

In order to prevent and treat gout, more than three times the diet should be done:

First, drink more water and drink less broth. For those with high serum uric acid and those with gout, drink more plain boiled water and less broth, fish broth, chicken broth, hotpot broth, etc. The osmolarity of plain boiled water is most conducive to dissolving various harmful substances in the body. A polydrink of plain boiled water can dilute uric acid and accelerate excretion, so that the uric acid level drops. The broth contains a large amount of purine components, which can not only dilute uric acid but rather lead to an increase in uric acid after drinking.

Secondly,eat more alkaline foods and less acidic foods. Patients with gout have purine metabolism disorders and abnormal uric acid, which can worsen the condition if they eat more acidic foods, and are not conducive to rehabilitation. However, eat more alkaline foods, which can help supplement potassium, sodium, and chloride ions to maintain acid-base balance, and the alkaline substances are not easily absorbed because of the patient's endocrine disorders. More soda containing minerals is recommended, a small amount of absorption is available below the action of trace minerals to benefit the improvement of the acidic environment in the patient.

Finally, eat more vegetables and less. Eat more vegetables, favor less purine intake, increase vitamin $C$, and increase cellulose. Less meals are beneficial for controlling caloric intake, weight restriction, and weight loss and lipid lowering.

\section{Conclusion}

It has been confirmed by studies that uric acid has a close relationship with kidney function [15]. Recently, with the development of research on the occurrence mechanism of kidney damage in hyperuricemia, its related aspects have received much attention from nephrologists. The current research focus on the damage mechanism of hyperuricemia is mainly focused on hyperuricemia indirectly damages the kidney, in addition, the vascular lesions caused by uric acid can also be manifested in increasing the viscosity of blood. Because uric acid belongs to the weak acid class and cannot be completely dissolved in plasma, upon temperature change this part is converted to become monosodium urate crystals. It awaits further investigation in multiple 
aspects due to the plethora of causative factors that trigger hyperuricemia.

\section{References}

1. Zhu Limei, Li Xianli. Research progress on the mechanism of hyperuricemia leading to abnormal glucose metabolism [J]. Medical review, 2019,25 (22): 4509-4514.

2. Zhao Hongjing, Yang yanlang, ye Yinyin, et al. Research progress of febuxostat in the treatment of hyperuricemia [J]. Anhui Medical Journal, 2018,39 (2): 239-241.

3. Lei Sanxia. Research progress on correlation between hyperuricemia and kidney damage [J]. Medical review, 2014,20 (10): 1775-1777.

4. Wang Hongyun. Research progress on pathogenesis of kidney damage in chronic hyperuricemia [J]. International Journal of Urology, 2011,31 (1): 109112.

5. Wang Yahua, Wang Yaping. Research progress of kidney damage in hyperuricemia [J]. Chinese Journal of evidence based cardiovascular medicine, 2013,5 (1): 91-92.

6. Wen Wen, Li Yuehong, Mu Rong. Research progress of kidney damage in hyperuricemia [J]. Journal of clinical internal medicine, 2016,33 (5): 309-311.

7. Huang Weiyuan. Efficacy observation and analysis of irbesartan plus amlodipine besylate in the treatment of elderly diabetes mellitus with hypertension and hyperuricemia $[\mathrm{J}]$. Chinese Journal of practical medicine, 2020,15 (2): 117-119.

8. Wang Tian, Chen Tao, Cai Qi, et al. Efficacy of febuxostat combined with potassium citrate sustained release tablets in the treatment of gout and hyperuricemia with kidney calculi $[\mathrm{J}]$. Journal of practical medicine, 2020,36 (2): 224-228.

9. Yin Xianglin, Yao Songpo, Li Xingzhou, et al. Risk factors of gout and hyperuricemia [J]. Chinese Journal of Gerontology, 2020,40 (10): 2041-2044.

10. Zhang Jingwen, Wang Ning, Qiu Hongxia, et al. Effect of febuxostat on kidney function in patients with early diabetic nephropathy complicated with hyperuricemia [J]. Shaanxi medical journal, 2020,49 (2): 209-211,230.

11. Xiao Xiaoqin, Chen shangru, Yao Chun, et al. A practical study on the intervention of multidisciplinary diet and exercise management in patients with hyperuricemia $[\mathrm{J}]$. Chinese and foreign medical research, 2020,18 (1): 158-160.

12. Liu Yuanhao. Clinical efficacy of Niaoduqing Granules Combined with febuxostat tablets in the treatment of hypertensive nephropathy with hyperuricemia [J]. Modern hospital, 2020,20 (3): 427-430434.

13. Dai Yuanhui, Hao Yuqi. Predictive significance of hyperuricemia for contrast induced nephropathy in
ACS patients with normal kidney function after PCI [J]. Chongqing Medical Journal, 2020,49 (20): 3427-3430.

14. Xiao Ni Shao, Mei Wei Wu, Ya Nan Hei, et al. Metabonomics of urine and kidney in rats with chronic hyperuricemic nephropathy $[\mathrm{J}]$. Chinese Pharmacological Bulletin, 2020,36 (11): 1607-1614.

15. Lu Zhenzhen, Deng Yueyi, Liu Wangyi. Correlation between hyperuricemia and $\operatorname{IgA}$ nephropathy in kidney biopsy [J]. Journal of Anhui Medical University, 2020,55 (3): 456-460. 